\title{
Extremal limit theorems for observations separated by random power law waiting times
}

\author{
Mark M. Meerschaert ${ }^{\mathrm{a}, *, 1}$, Stilian A. Stoev ${ }^{\mathrm{b}, 2}$ \\ ${ }^{a}$ Department of Statistics and Probability, Michigan State University, East Lansing, MI 48824, USA \\ ${ }^{\mathrm{b}}$ Department of Statistics, University of Michigan, Ann Arbor, MI 48109, USA
}

\section{A R T I C L E I N F O}

\section{Article history:}

Received 27 March 2008

Received in revised form

23 September 2008

Accepted 9 October 2008

Available online 21 October 2008

MSC:
60G70
60F15
Keywords:
Extremal process
Renewal process
Subordinator

\begin{abstract}
A B S T R A C T
This paper develops extreme value theory for random observations separated by random waiting times whose exceedence probability falls off like a power law. In the case where the waiting times between observations have an infinite mean, a limit theorem is established, where the limit is comprised of an extremal process whose time index is randomized according to the non-Markovian hitting time process for a stable subordinator. The resulting limit distributions are shown to be solutions of fractional differential equations, where the order of the fractional time derivative coincides with the power law index of the waiting time. The probability that the limit process remains below a threshold is also computed. For waiting times with finite mean but infinite variance, a two-scale argument yields a fundamentally different limit process. The resulting limit is an extremal process whose time index is randomized according to the first passage time of a positively skewed stable Lévy motion with positive drift. This two-scale limit provides a second-order correction to the usual limit behavior.
\end{abstract}

(c) 2008 Elsevier B.V. All rights reserved.

\section{Introduction}

Classical extreme value theory assumes that observations are collected at regular intervals, or at non-random points in time. In some applications, waiting times between observations (or between observations that exceed a given threshold) are heavy tailed. For waiting times with regularly varying probability tails, resembling a power law, the mean waiting time or the variance of the waiting times can be infinite. For waiting times with infinite mean, the renewal process that counts the number of observations by time $t$ grows at a sublinear rate, and the renewal theorem does not apply (see, e.g., Feller, 1971, XI.5). For the intermediate case, where the waiting times between observations have a finite mean but infinite variance, the renewal process is asymptotically constant, but the convergence to this constant is rather slow. Hence it is advantageous to apply a second-order correction in the limit theory. This paper develops limit theory for both cases, and establishes explicit distributional formulae for the limit process. It also provides a concrete expression for the probability that the limit process remains below a threshold, using a transformation formula for stochastic integrals with respect to a subordinator.

Power law waiting times are common in many applications. In computer science, Resnick and Stărică (1995) show that quiet periods $J$ between transmissions for a networked computer terminal have a heavy tail with $P(J>t) \approx C t^{-\beta}$ where $\beta \approx 0.6$.

\footnotetext{
* Corresponding author. Tel.: +1517432 7097.

E-mail addresses: mcubed@stt.msu.edu (M.M. Meerschaert), sstoev@umich.edu (S.A. Stoev).

${ }^{1}$ This author was partially supported by NSF Grant DMS-0706440.

2 This author was partially supported by NSF Grant DMS-0806094.
} 
In finance, Mainardi et al. (2000) show that waiting times between trades of certain bond futures have a heavy tail with $\beta \approx 0.95$. Barabási (2005) reports $\beta \approx 1$ for waiting time between emails, and for waiting time for a reply. Berhondo et al. (2006) estimate $1<\beta<2$ for waiting times between coronal mass ejections (solar flares). Brockmann et al. (2006) obtain $\beta=1.05$ as the waiting time for paper currency to travel beyond the limits of a metropolitan area. They also report a power law distribution in the ensuing travel distance. Cheng et al. (1995) observe $\beta=1.6$ in the waiting time between earthquakes, and a similar value for waiting time between 'starquakes' in neutron stars. Lavergnat and Golé (1998) measured power law waiting times with $\beta=0.68$ between large raindrops. Smethurst and Williams (2001) find $\beta=1.4$ in the waiting times for a doctor appointment. Sabatelli et al. (2002) report $\beta=0.4$ for 19 th century Irish stock prices, and $\beta=1.87$ for the modern Japanese Yen currency market. Benson et al. (2007) discuss the important implications of random waiting times between observations, for extreme values in geophysics.

In this paper, we develop limit theorems for extremes with heavy tailed random waiting times between observations. This involves studying the limit behavior of maxima of random number of terms. Many authors have contributed to the asymptotic theory of sums, maxima, and general random sequences with a random number of terms. See, for example, Korolev (1994, 1995), the books of Kruglov and Korolev (1990), Bening and Korolev (2002), and the references therein. Here, however, our focus is on convergence of processes and their connections to fractional calculus. Limit theorems for extremal processes with random waiting times were considered by Resnick (1986), who discusses the case where the number of observations forms a random walk. Our case differs, since the number of observations here is driven by a renewal process, which is the inverse of that random walk. Silvestrov and Teugels $(1998,2004)$ develop a general theory for the joint behavior of sums and maxima in random observation times. Pancheva and Jordanova (2004) specifically consider the case of infinite mean waiting times $(0<\beta<1)$, by adapting arguments in Meerschaert and Scheffler (2004), along with a powerful transfer theorem (Silvestrov and Teugels, 1998, Theorem 3). Pancheva (1998) explores self-similar extremal processes, and random time-changed extremal processes are also considered in Pancheva et al. (2006a, b). Our Theorem 3.1 is closely related to the results of Pancheva and Jordanova (2004) and Silvestrov and Teugels (2004). For waiting times with finite mean but infinite variance, classical renewal theory shows that the number of observations by time $t$ grows at a linear rate, and the renewal rate is asymptotically constant. This leads to a well-known limit result that involves a simple change of scale in time for the extremal process (see, e.g., Resnick, 1986). For heavy tail waiting times, convergence in the renewal theorem is slower than in the finite variance case, and thus it is advantageous to make a second-order correction. This is accomplished using the two-scale limit procedure introduced in Becker-Kern et al. (2003) and Meerschaert and Scheffler (2004) for random walk models.

Fractional derivatives are almost as old as their more familiar integer-order counterparts Miller and Ross (1993) and Samko et al. (1993). Fractional differential equations are useful to model various problems in physics (see a comprehensive review by Metzler and Klafter, 2004), finance (see a recent review by Scalas, 2006), and hydrology (Baeumer et al., 2001; Benson et al., 2001, 2000; Schumer et al., 2001). Time-fractional derivatives are connected with physical models of particle sticking and trapping (Meerschaert et al., 2002). For events separated by waiting times with whose exceedence probability belongs to some sum-stable domain of attraction, a connection between limit theorems and fractional time derivatives was laid out in Becker-Kern et al. (2003) and Meerschaert and Scheffler (2004). Specifically, we assume that the exceedence probability is regularly varying; this means, essentially, that the probability of waiting longer than time $t>0$ falls off like a power law $t^{-\beta}$. Then it turns out that the governing equation for the cumulative probability distribution functions for the limit process solve a fractional ordinary differential equation, where the order $\beta$ of the time derivative equals the power law index of the waiting times.

This paper develops limit theorems for maxima of observations separated by random waiting times, by covering two substantially different scenarios:

(i) infinite mean waiting times $(0<\beta<1)$;

(ii) finite mean but infinite variance waiting times $(1<\beta<2)$.

The limit process in case (i) is an extremal process whose time index is randomized via the non-Markovian hitting time process of a stable subordinator with index $0<\beta<1$. In this case, we also provide a new formula for the probability that the randomized process remains below a given threshold function, which is important for applications in insurance, hydrology, and telecommunications (see, e.g., Pancheva et al., 2007; Lavergnat and Golé, 1998; Barabási, 2005). In case (ii), where the mean of the waiting times is finite, the limit process is an extremal process whose time index is randomized according to the first passage time process of a stable Lévy motion with index $1<\beta<2$ and positive drift depending on the mean waiting time. Our result stands in contrast to established results in the literature, because we consider a novel two-scale limit scenario. Limit theorems for continuous time random walks, where the waiting time between jumps is separated by random waiting times with heavy tails, were developed in Becker-Kern et al. (2003) and Meerschaert and Scheffler (2004). In the present study, we adapt the method used there for the waiting time process, replacing the sum in those papers with the maximum. The boundary regime when $\beta=1$ is delicate, and will be explored in a future work.

The paper is organized as follows. Section 2 lays out our basic assumptions, in order to establish notation. Section 3 presents a limit theorem for the case of infinite-mean waiting times $(0<\beta<1)$. This leads to fundamental connections to fractional calculus. Some applications, including the time the limit process from Section 3 spends below a given threshold function, are discussed in Section 4. Section 5 addresses the case of finite-mean waiting times $(1<\beta<2)$ from the perspective of a two-scale asymptotic regime. Finally, some auxiliary results are collected in Section 6. 


\section{Problem formulation and basic results}

Let $J_{1}, J_{2}, \ldots$ be non-negative independent and identically distributed (i.i.d) random variables that model the waiting times between observations. We set $T(0)=0$ and $T(n)=\sum_{j=1}^{n} J_{j}$, the time of the $n$th jump. The observations are modeled by i.i.d. random variables $Y_{1}, Y_{2}, \ldots$ on $\mathbb{R}$ which are assumed independent of $\left(J_{i}\right)$. Let $M_{0}=0$ and $M_{Y}(n)=\max \left\{Y_{i}: i=1, \ldots, n\right\}$, the maximum of the first $n$ observations. For $t \geqslant 0$ let

$$
N_{t}=\max \{n \geqslant 0: T(n) \leqslant t\}
$$

the number of observations by time $t$, and define

$$
X(t)=M_{Y}\left(N_{t}\right)=\max \left\{Y_{i}: i=1, \ldots, N_{t}\right\}
$$

the maximum observed by time $t$.

Assume that $J_{1}$ belongs to the strict sum-domain of attraction of some stable law with index $0<\beta<1$. This means that there exist $b_{n}>0$ such that

$$
b_{n}\left(J_{1}+\cdots+J_{n}\right) \Rightarrow D
$$

where $D>0$ almost surely. Here $\Rightarrow$ denotes convergence in distribution. The distribution $\rho$ of $D$ is stable with index $\beta$, meaning that $\rho^{t}=t^{1 / \beta} \rho$ for all $t>0$, where $\rho^{t}$ is the $t$-th convolution power of the infinitely divisible law $\rho$ and $(a \rho)\{\mathrm{d} x\}=\rho\left\{a^{-1} \mathrm{~d} x\right\}$ is the probability distribution of $a D$ for $a>0$. Moreover $\rho$ has a Lebesgue density $g_{\beta}$ which is a $C^{\infty}$-function.

For $t \geqslant 0$ let $T(t)=\sum_{j=1}^{[t]} J_{j}$ and let $b(t)=b_{[t]}$, where [t] denotes the integer part of $t$. Then $b(t)=t^{-1 / \beta} L(t)$ for some slowly varying function $L(t)$ (so that $L(\lambda t) / L(t) \rightarrow 1$ as $t \rightarrow \infty$ for any $\lambda>0$, see for example Feller, 1971) and it follows from Example 11.2.18 of Meerschaert and Scheffler (2001) that

$$
\{b(c) T(c t)\}_{t} \geqslant 0 \stackrel{f d d}{\Longrightarrow}\{D(t)\}_{t} \geqslant 0 \quad \text { as } c \rightarrow \infty
$$

where $\stackrel{f d d}{\Longrightarrow}$ denotes convergence in distribution of all finite dimensional marginal distributions. Then $\{D(t)\}$ is a strictly stable Lévy process that is self-similar with exponent $H=1 / \beta>1$, that is,

$$
\{D(c t)\}_{t \geqslant 0} \stackrel{f d d}{=}\left\{c^{1 / \beta} D(t)\right\}_{t} \geqslant 0
$$

for all $c>0$, where $\stackrel{f d d}{=}$ denotes equality of all finite dimensional marginal distributions. Note that by Example 21.7 of Sato (1999) the sample paths of $\{D(t)\}$ are almost surely increasing. Moreover, since $D(t) \stackrel{d}{=} t^{1 / \beta} D$, where $\stackrel{d}{=}$ means equal in distribution, it follows that

$$
D(t) \rightarrow \infty \text { in probability as } t \rightarrow \infty
$$

Then it follows from Theorem I.19 in Bertoin (1996) that $D(t) \rightarrow \infty$ almost surely as $t \rightarrow \infty$.

The sample paths of the Lévy motion $\{D(x)\}_{x \geqslant 0}$ are continuous from the right, with left-hand limits, strictly increasing, and $D(0)=0$ and $D(x) \rightarrow \infty$ as $x \rightarrow \infty$. Hence the hitting time process

$$
E(t)=\inf \{x: D(x)>t\}
$$

is well defined. These inverse processes are related by

$$
\{E(t) \leqslant x\}=\{D(x) \geqslant t\}
$$

for all $x>0$ and $t>0$. Proposition 3.1 in Meerschaert and Scheffler (2004) shows that the process $\{E(t)\}_{t \geqslant 0}$ defined by $(2.7)$ is self-similar with exponent $\beta \in(0,1)$, that is for any $c>0$ we have

$$
\{E(c t)\}_{t \geqslant 0} \stackrel{f d d}{=}\left\{c^{\beta} E(t)\right\}_{t} \geqslant 0
$$

We also have $E(t) \stackrel{d}{=}(D / t)^{-\beta}$ for any $t>0$, where $D$ is from (2.3), and it follows that $E(t)$ has moments of all orders. Furthermore, the random variable $E(t)$ has density

$$
f_{t}(s)=\frac{t}{\beta} s^{-1-1 / \beta} g_{\beta}\left(t s^{-1 / \beta}\right),
$$

where $g_{\beta}$ is the density of the limit $D$ in (2.3), see Meerschaert and Scheffler (2004, Corollary 3.1). The process $\{E(t)\}_{t} \geqslant 0$ has neither stationary nor independent increments, but has almost surely continuous sample paths, and hence is continuous in probability. 
Recall that the function $b$ in (2.4) is regularly varying with index $-1 / \beta$. Hence $1 / b$ is regularly varying with index $1 / \beta>0$ so by Property 1.5 .5 of Seneta (1976) there exists a regularly varying function $\tilde{b}$ with index $\beta$ such that $1 / b(\tilde{b}(c)) \sim c$ as $c \rightarrow \infty$. Here we use the notation $f \sim g$ for positive functions $f, g$ if and only if $f(c) / g(c) \rightarrow 1$ as $c \rightarrow \infty$. Equivalently we have

$$
b(\tilde{b}(c)) \sim \frac{1}{c} \quad \text { as } c \rightarrow \infty
$$

Theorem 3.2 in Meerschaert and Scheffler (2004) shows that

$$
\left\{\tilde{b}(c)^{-1} N_{c t}\right\} \stackrel{f d d}{\Longrightarrow}\{E(t)\}_{t \geqslant 0} \quad \text { as } c \rightarrow \infty
$$

and Meerschaert and Scheffler (2004, Corollary 3.3) shows that the convergence also holds in the Skorohod $\operatorname{space} D([0, \infty)$, [0, $\infty)$ ) with the $J_{1}$-topology.

Assume that $\left(Y_{i}\right)$ are i.i.d. $\mathbb{R}$-valued random variables independent of $\left(J_{i}\right)$ and assume that $Y_{1}$ belongs to the max-domain of attraction of some non-degenerate max-stable random variable $A$ with distribution function $F(x)=P\{A \leqslant x\}$. Then there exist sequences $a_{n}$ and $d_{n}$ such that

$$
a_{n}\left(M_{Y}(n)-d_{n}\right)=a_{n}\left(\max \left\{Y_{i}: i=1, \ldots, n\right\}-d_{n}\right) \Rightarrow A \quad \text { as } n \rightarrow \infty
$$

Define the extremal process $\{A(t)\}_{t>0}$ as in Dwass (1964) (see also Resnick, 1987) so that $P\{A(t) \leqslant x\}=F(x)^{t}$ for all $t>0$, and in fact, for all $0<t_{1}<\ldots<t_{d}$ and $x_{i} \in \mathbb{R}, 1 \leqslant i \leqslant d$,

$$
P\left\{A\left(t_{i}\right) \leqslant x_{i}, 1 \leqslant i \leqslant d\right\}=F\left(\min _{1 \leqslant i \leqslant d} x_{i}\right)^{t_{1}} F\left(\min _{2 \leqslant i \leqslant d} x_{2}\right)^{t_{2}-t_{1}} \cdots F\left(x_{d}\right)^{t_{d}-t_{d-1}} .
$$

We now define the continuous-time partial maxima process $\left\{M_{Y}(t)\right\}_{t>0}$ as

$$
M_{Y}(t)= \begin{cases}\max \left\{Y_{i}: i=1, \ldots,[t]\right\}, & t \geqslant 1 \\ Y_{1}, & 0<t<1\end{cases}
$$

By Theorem 3.2 in Lamperti (1964) (see also Resnick, 1975, Theorem 2), we have

$$
\left\{a(c)\left(M_{Y}(c t)-d(c)\right)\right\}_{t>0} \Rightarrow\{A(t)\}_{t>0} \quad \text { as } c \rightarrow \infty,
$$

in the Skorohod space $D((0, \infty), \mathbb{R})$ of càdlág (right continuous with left-hand limits) functions defined on $(0, \infty)$ and equipped with the $J_{1}$-topology, where $a(t)=a_{[t]}, d(t)=d_{[t]}$.

We note that up to a scale or location parameter, the extreme value distribution $F(x)$ can take one of the three standard forms

$$
\begin{aligned}
& \text { Gumbel : } \Lambda(x)=\mathrm{e}^{-\mathrm{e}^{-x}} \text { for } x \in \mathbb{R}, \\
& \text { Fréchet : } \Phi_{\alpha}(x)=\mathrm{e}^{-x^{-\alpha}} \text { for } x \geqslant 0, \\
& \text { reversed-Weibull (or negative Fréchet): } \Psi_{\alpha}(x)=\mathrm{e}^{-(-x)^{\alpha}} \quad \text { for } x \leqslant 0,
\end{aligned}
$$

where $\alpha$ is some positive constant.

The limit $F$-extremal processes $\{A(t)\}_{t>0}$ have non-decreasing paths and are always continuous in probability (Resnick and Rubinovitch, 1973, Proposition 1). Their behavior near $t \approx 0$ and as $t \rightarrow \infty$, however, differs depending on the type of the limit distribution $F$.

(Gumbel) If $F(x)=\Lambda(x)$, then $A(t) \rightarrow \infty$, almost surely, as $t \rightarrow \infty$ and also $A(t) \rightarrow-\infty$, almost surely, as $t \downarrow 0$ (see also Resnick, 1974, Theorem 7 for more details).

(Fréchet) If $F(x)=\Phi_{\alpha}(x)$, then $A(t) \rightarrow \infty$, almost surely, as $t \rightarrow \infty$, but now $A(t) \rightarrow 0$, almost surely, as $t \downarrow 0$. In this case, one can take $d(c)=0$ in (2.14) and $a(c) \rightarrow 0$, as $c \rightarrow \infty$.

(reversed-Weibull) If $F(x)=\Psi_{\alpha}(x)$, then $A(t) \rightarrow 0$, almost surely, as $t \rightarrow \infty$ and $A(t) \rightarrow-\infty$, almost surely, as $t \downarrow 0$. Now, the centering $d(c)$ in (2.14) depends on the distribution of $Y_{i}$ and $a(c) \rightarrow \infty$ as $c \rightarrow \infty$.

For detailed examples of possible normalizations $a(c)$ and $d(c)$ occurring in each one of the above three cases, see e.g., Leadbetter et al. (1983, Chapter 1.7).

Remark 2.1. In the case of Fréchet marginals one can define the extremal process $\{A(t)\}$ at $t=0$ as zero. In the other two cases, the process $A$ diverges to $-\infty$ as $t \downarrow 0$ and it cannot be defined at $t=0$. 


\section{Infinite mean waiting times: limit theorems and fractional governing equations}

In this section, we first present a functional limit theorem for the process $\{X(t)\}$ defined in (2.2) under the distributional assumptions of Section 2. The limiting process $\{M(t)\}_{t>0}$ is a randomization or mixing of the extremal process $\{A(t)\}_{t>0}$ in $(2.14)$ by the hitting time process $\{E(t)\}_{t>0}$ introduced in Section 2 . We then establish a fundamental relationship between the distribution of the limiting process $\{M(t)\}_{t>0}$ and fractional ordinary differential equations.

Recall from the paragraph before (2.10) that $\tilde{b}$ is regularly varying with index $\beta$. We define $\tilde{a}(c)=a(\tilde{b}(c))$ and $\tilde{d}(c)=d(\tilde{b}(c))$. The following result has already implicitly appeared in the literature (see e.g., Silvestrov and Teugels, 2004, Theorem 3.1; Pancheva and Jordanova, 2004, Theorem 4). We arrived at it independently. For completeness, we give here a simple proof in our notation by using the results of Silvestrov (2004).

Theorem 3.1. Under the assumptions of Section 2 we have

$$
\{\tilde{a}(c)(X(c t)-\tilde{d}(c))\}_{t>0} \Rightarrow\{M(t)\}_{t>0} \quad \text { in } D((0, \infty), \mathbb{R}) \text { as } c \rightarrow \infty
$$

in the $J_{1}$-topology, where $\{M(t)\}_{t>0}=\{A(E(t))\}_{t>0}$ is a mixture process.

Proof. Note that since $\left(J_{i}\right)$ and $\left(Y_{i}\right)$ are independent, the processes $\left\{M_{Y}(t)\right\}_{t>0}$ and $\left\{N_{t}\right\}_{t>0}$ in (2.2) are independent. Hence it follows from (2.14) and Corollary 3.4 in Meerschaert and Scheffler (2004) that we also have

$$
\left\{\left(\tilde{a}(c)\left(M_{Y}(\tilde{b}(c) t)-\tilde{d}(c)\right), \tilde{b}(c)^{-1} N_{c t}\right)\right\}_{t>0} \Rightarrow\{(A(t), E(t))\}_{t>0} \quad \text { as } c \rightarrow \infty
$$

in $D((0, \infty), \mathbb{R}) \times D((0, \infty),(0, \infty))$ in the $J_{1}$-topology. The limit processes $A=\{A(t)\}_{t>0}$ and $E=\{E(t)\}_{t>0}$ are independent and continuous in probability. Since, in addition, $E$ has continuous sample paths, by Silvestrov $(2004$, Theorem 3.4 .1$)$, the convergence in (3.2) implies the convergence in (3.1) (recall (2.2)).

Remark 3.2. An alternative proof of Theorem 3.1 can be obtained as in Pancheva and Jordanova (2004, Theorem 4), using results from Meerschaert and Scheffler (2004) along with the transfer theorem of Silvestrov and Teugels (1998). One can also prove Theorem 3.1 by following the proof of Theorem 4.1 in Becker-Kern et al. (2003). Indeed, by using a transfer type theorem one can first show that (3.1) holds in the sense of finite-dimensional distributions. This, by monotonicity, yields convergence in the $J_{1}$-topology (Bingham, 1971, Theorem 3).

Recall that the distribution $P\{A(t) \leqslant x\}=F(x)^{t}$ and that $E(t)$ has density $f_{t}(s)$ given by (2.9) where $g_{\beta}$ is the density of the limit $D$ in (2.3). Then, since $\{A(t)\}_{t>0}$ and $\{E(t)\}_{t>0}$ are independent stochastic processes, it follows from a simple conditioning argument that for any $t>0$ we have

$$
\begin{aligned}
P\{M(t) \leqslant x\} & =\int_{0}^{\infty} P\{A(s) \leqslant x\} f_{t}(s) \mathrm{d} s \\
& =\int_{0}^{\infty} F(x)^{s} f_{t}(s) \mathrm{d} s=\frac{t}{\beta} \int_{0}^{\infty} F(x)^{s} s^{-1-1 / \beta} g_{\beta}\left(t s^{-1 / \beta}\right) \mathrm{d} s .
\end{aligned}
$$

We now give some intuition as to the nature of the limit process $M(t)=A(E(t))$ by deriving its marginal distribution in the case of a heavy-tailed extremal process $\{A(t)\}_{t>0}$, which is of primary interest in our applications.

Suppose that the observations $\left(Y_{i}\right)$ satisfy $P\left\{Y_{i}>x\right\} \sim C_{1} x^{-\alpha}$ as $x \rightarrow \infty$ for some $\alpha>0$ and $C_{1}>0$. Then we can take $a_{n}=n^{-1 / \alpha}$ and $d(c)=0$ and we get convergence (2.12) with $F(x)=P\{A \leqslant x\}=\mathrm{e}^{-C_{1} x^{-\alpha}}$ for $x>0$. Suppose also that the waiting times $\left(J_{i}\right)$ satisfy $P\left\{J_{i}>t\right\} \sim C_{2} t^{-\beta}$ as $t \rightarrow \infty$ for some $0<\beta<1$ and $C_{2}>0$. Then we can take $b_{n}=\left(C_{2} n\right)^{-1 / \beta}$, with $\tilde{b}(c)=c^{\beta} / C_{2}$ (see $\left.(2.10)\right)$ and we get convergence (2.3) where $\mathbb{E}\left(\mathrm{e}^{-s D}\right)=\mathrm{e}^{-\Gamma(1-\beta) s^{\beta}}$ (see Lemma 6.3). Now $\tilde{a}(c)=C_{2}^{1 / \alpha} c^{-\beta / \alpha}$, leading to $\left(C_{2}^{-1 / \beta} c\right)^{-\beta / \alpha} X(c t) \Rightarrow M(t)$ for all $t>0$ as $c \rightarrow \infty$. Recall that $X(c t)$ is the largest observation by time $c t$ where $c$ represents a time scale. Then we can approximate $X(c t) \approx\left(C_{2}^{-1 / \beta} c\right)^{\beta / \alpha} M(t)$ for $c$ large (long time scale) where

$$
P\{M(t) \leqslant x\}=\frac{t}{\beta} \int_{0}^{\infty} \mathrm{e}^{-s C_{1} x^{-\alpha}} s^{-1-1 / \beta} g_{\beta}\left(t s^{-1 / \beta}\right) \mathrm{d} s .
$$

Here $g_{\beta}$ is the density of the stable subordinator $D$, a stable law with index $\beta$, skewness equal to one, center $\mu=0$ and scale $\sigma^{\beta}=\Gamma(1-\beta) \cos (\pi \beta / 2)$ in the standard parameterization of Samorodnitsky and Taqqu (1994, Definition 1.1.6). (Note that $\beta$ here denotes the index of stability and not the skewness of $D$.) It follows that

$$
\begin{aligned}
P\{X(c t) \leqslant x\} & \approx P\left\{\left(C_{2}^{-1 / \beta} c\right)^{\beta / \alpha} M(t) \leqslant x\right\}=P\left\{M\left(C_{2}^{-1 / \beta} c t\right) \leqslant x\right\} \\
& =\frac{C_{2}^{-1 / \beta} c t}{\beta} \int_{0}^{\infty} \mathrm{e}^{-s C_{1} x^{-\alpha}} s^{-1-1 / \beta} g_{\beta}\left(C_{2}^{-1 / \beta} c t s^{-1 / \beta}\right) \mathrm{d} s
\end{aligned}
$$

when the time scale $c>0$ is sufficiently large. 
Observe that the scale $C_{2}$ of the waiting times $J_{i}$ 's and the time scale $c$ are inversely related. Indeed, the larger the time scale $c$, the more time has elapsed and hence, the larger the values of $X(c t)$. On the other hand, the larger the scale $C_{2}$, the longer the waiting times, and hence the smaller the values of $X(c t)$.

We discuss next the fundamental connection between the limit process $M(t)=A(E(t))$ in Theorem 3.1 and fractional calculus. The extreme value distributions (2.15) are all of the form $\Lambda(x)=\mathrm{e}^{-a(x)}$ where $a(x)$ takes one of the three forms depending on the type. The extremal process $A(t)$ therefore has distribution function $F_{t}(x)=\mathrm{e}^{-t a(x)}$ and obviously this function solves the ordinary differential equation

$$
\frac{\partial}{\partial t} F_{t}(x)=-a(x) F_{t}(x), \quad F_{0}(x)=\phi_{a(\cdot)}(x)
$$

where

$$
\phi_{a(\cdot)}(x)= \begin{cases}I(x \geqslant 0) & \text { if } a(x)=I(x \geqslant 0) x^{-\alpha} \\ 1 & \text { if } a(x)=I(x<0)(-x)^{\alpha} \text { or } a(x)=\mathrm{e}^{-x} .\end{cases}
$$

The initial conditions $F_{0}(x)=\phi_{a(\cdot)}(x)$ for the Cauchy problem in (3.5) depend on the type of the extreme value distribution. For example, in the situation of (3.4) we have $a(x)=C_{1} x^{-\alpha}$ for some $C_{1}>0$ and $\alpha>0$, and $F_{0}(x)$ is the Heaviside function, i.e., a probability distribution with a unit mass at the origin. On the other hand, in the other two cases of extreme value distributions (reversed-Weibull and Gumbel), $F_{0}(x) \equiv 1$ can be interpreted as the probability distribution with a unit mass at $-\infty$. The next result gives the analogous governing equation for the limit process $M(t)$ in Theorem 3.1 .

The Caputo fractional derivative of order $0<\beta<1$ is defined by

$$
\frac{\mathrm{d}^{\beta}}{\mathrm{d} t^{\beta}} f(t)=\frac{1}{\Gamma(1-\beta)} \int_{0}^{t} f^{\prime}(t-u) u^{-\beta} \mathrm{d} u
$$

where $\Gamma(\cdot)$ is the usual Gamma function and $f^{\prime}(t)$ is the first derivative. Writing $\tilde{f}(\lambda)=\int_{0}^{\infty} \mathrm{e}^{-\lambda t} f(t) \mathrm{d} t$ for the standard Laplace transform, the Caputo fractional derivative $\mathrm{d}^{\beta} f(t) / \mathrm{d} t^{\beta}$ has Laplace transform

$$
\int_{0}^{\infty} \mathrm{e}^{-\lambda t} \frac{\mathrm{d}^{\beta}}{\mathrm{d} t^{\beta}} f(t) \mathrm{d} t=\lambda^{\beta} \tilde{f}(\lambda)-\lambda^{\beta-1} f(0), \quad \lambda \in \mathbb{R}
$$

extending the well-known formula for the integer-order derivative $\beta=1$. See for example Caputo (1967) and Podlubny (1999).

Theorem 3.3. Under the assumptions of Theorem 3.1, the distribution function $G_{t}(x)=P\{M(t) \leqslant x\}$ of the limit process solves the governing ordinary differential equation

$$
\frac{\partial^{\beta}}{\partial t^{\beta}} G_{t}(x)=-a(x) G_{t}(x), \quad G_{0}(x)=\phi_{a(\cdot)}(x),
$$

where the Caputo fractional derivative appears on the left-hand side, $\phi_{a(\cdot)}(x)$ is as in (3.6), and $a(x)$ is the exponent of the extremal limit process $A(t)$, so that $A(t)$ has distribution function $F_{t}(x)=\mathrm{e}^{-t a(x)}$ for all $t>0$.

Proof. From (3.3) we have

$$
G_{t}(x)=\int_{0}^{\infty} \mathrm{e}^{-s a(x)} f_{t}(s) \mathrm{d} s
$$

where $f_{t}(s)$ is the density of $E(t)$. Theorem 3.1 in Meerschaert and Scheffler (2008) shows that $f_{t}(s)$ has Laplace transform $t \mapsto \lambda$ given by $\lambda^{\beta-1} \mathrm{e}^{-s \lambda^{\beta}}$ (take $\psi_{D}(\lambda)=\lambda^{\beta}$ in (3.13) of that paper). Hence a simple Tonelli-Fubini argument shows that the Laplace transform

$$
\tilde{G}_{\lambda}(x)=\int_{0}^{\infty} \mathrm{e}^{-s a(x)} \lambda^{\beta-1} \mathrm{e}^{-s \lambda^{\beta}} \mathrm{d} s=\frac{\lambda^{\beta-1}}{\lambda^{\beta}+a(x)}
$$

which leads to

$$
\lambda^{\beta} \tilde{G}_{\lambda}(x)-\lambda^{\beta-1}=-a(x) \tilde{G}_{\lambda}(x) .
$$

Invert the Laplace transform using Eq. (3.8) to obtain (3.9). Note that $G_{0}(0)=1$ in all cases of extreme value distributions. Note also that $G_{t}(x)$ is continuous in $t>0$ since the process $M(t)=A(E(t)), t>0$ is continuous in probability (Lemma 6.1). Therefore, for each $x$, the Laplace inversion yields a unique classical solution on $t>0$ for each $x$. See, for example, Feller (1971, p. 431). 
Remark 3.4. It may also be possible to prove Theorem 3.3 by appealing to more general results such as Theorem 3.1 in Baeumer and Meerschaert (2001). However, this requires establishing the appropriate semigroup conditions in a functional analytic setting, which is tedious in the case of distribution functions.

Remark 3.5. The connection between hitting time densities for stable subordinators and Mittag-Leffler functions was noted by Bingham (1971) and Bondesson et al. (1996). Inverting the Laplace transform (3.10) we immediately obtain an alternative form of the limit distribution function:

$$
G_{t}(x)=E_{\beta}\left(-a(x) t^{\beta}\right)
$$

in terms of the Mittag-Leffler function defined by

$$
E_{\beta}(z)=\sum_{k=0}^{\infty} \frac{z^{k}}{\Gamma(1+\beta k)},
$$

see, for example, Mainardi and Gorenflo (2000).

\section{A transformation formula with applications to durations}

Here, we give a transformation formula linking a monotone non-decreasing càdlág function $D=\{D(x)\}_{x} \geqslant 0$ with $D(0)=0$ and its (right-continuous) inverse $E=\{E(t)\}_{t \geqslant 0}$. We then use this result to derive the distribution of the duration the process $M(t)=A(E(t))$ spends below a given threshold $a>0$, where $\{E(t)\}$ is the inverse of a $\beta$-stable subordinator $(0<\beta<1)$. This result has important applications in risk management.

Lemma 4.1. Let $E(t)=\inf \{x \geqslant 0: D(x)>t\}, t \geqslant 0$ for a non-decreasing càdlág function $D=\{D(x)\}_{x} \geqslant 0$ with $D(0)=0$. Then for any non-negative Borel measurable $f:[0, \infty) \rightarrow[0, \infty)$, we have

$$
\int_{(0, \infty)} f(E(t)) \mathrm{d} t=\int_{(0, \infty)} f(x) \mathrm{d} D(x)
$$

where the first is a Lebesgue and the second a Lebesgue-Stieltjes integral.

Proof. One can show using (2.8) that, for all $a>0$

$$
D(a)=\inf \{t \geqslant 0: E(t)>a\}=\sup \{t \geqslant 0: E(t) \leqslant a\} .
$$

Since $\{E(t)\}$ is monotone and non-decreasing, the last supremum equals $\int_{(0, \infty)} \llbracket(E(t) \leqslant a) \mathrm{d} t$. This implies that

$$
D(b)-D(a)=\int_{(0, \infty)} \mathbb{q}_{(a, b]}(E(t)) \mathrm{d} t \text { for all } 0<a<b
$$

Let $\mu_{D}(a, b]:=D(b)-D(a)$ for all $0<a<b$ be the (random) Lebesgue-Stieltjes measure corresponding to the function $D$ and consider the occupation measure $\mu_{E}(B):=\int_{(0, \infty)} \rrbracket_{B}(E(t)) \mathrm{d} t$, for any Borel set $B \subset(0, \infty)$. Relation (4.2) implies that the $\sigma$-finite measures $\mu_{D}$ and $\mu_{E}$ agree on the semiring generated by $\{(a, b], 0<a<b\}$, and hence they agree on all Borel sets. This implies (4.1) for any Borel measurable function $f \geqslant 0$. Note that $f(E(t))$ is Borel measurable since $E$ is monotone.

An application to durations: Let $a>0$ and let

$$
\xi_{a}:=\sup \{t: M(t) \leqslant a\}=\int_{0}^{\infty} \mathbb{q}_{\{M(t) \leqslant a\}} \mathrm{d} t
$$

where $M(t)=A(E(t))$ is the mixture $F$-extremal process in Theorem 3.1. The random variable $\xi_{a}$ is the first hitting time of level $a$ for the process $M$. It may also be interpreted as the duration the process spends below the threshold $a$.

Proposition 4.2. For the random variable $\xi_{a}$ in (4.3), we have

$$
\xi_{a} \stackrel{d}{=}(-\log F(a))^{-1 / \beta} X^{1 / \beta} D(1)
$$

where $X$ is an Exponential random variable with mean one, independent of $D$. 
Proof. We have

$$
\xi_{a}=\int_{0}^{\infty} \mathbb{\square}_{\{M(t) \leqslant a\}} \mathrm{d} t=\int_{0}^{\infty} \mathbb{q}_{\{A(E(t)) \leqslant a\}} \mathrm{d} t=\int_{0}^{\infty} f(E(t)) \mathrm{d} t,
$$

where $f(x):=\rrbracket_{\{x: A(x) \leqslant a\}}$. By the independence of the processes $A$ and $D$, Lemma 4.1 implies that

$$
\xi_{a}=\int_{0}^{\infty} f(E(t)) \mathrm{d} t=\int_{0}^{\infty} f(x) \mathrm{d} D(x) \text { almost surely. }
$$

By Lemma 6.2, the last integral may be viewed in the sense of Samorodnitsky and Taqqu (1994, Chapter 3), as a stochastic integral with respect to an independently scattered stable random measure. However, since $f$ is independent of $D$, by conditioning, we obtain

$$
\int_{0}^{\infty} f(x) \mathrm{d} D(x) \stackrel{d}{=}\left(\int_{0}^{\infty}|f(x)|^{\beta} \mathrm{d} x\right)^{1 / \beta} D(1)=\left(\int_{0}^{\infty} 1_{\{x: A(x) \leqslant a\}} \mathrm{d} x\right)^{1 / \beta} D(1) .
$$

Observe that $\int_{0}^{\infty} \mathbb{q}_{\{x: A(x) \leqslant a\}} \mathrm{d} x$ is the first hitting time of level $a$ for the F-extremal process $A$. As shown in Resnick (1974, pp. 394-395), this hitting time is exponential with mean $1 /(-\log F(a))$. Therefore, the right-hand side of (4.4) equals $(-\log F(a))^{-1 / \beta} X^{1 / \beta} D(1)$, where $X$ is a standard exponential variable, independent of $D$.

We address next the following problem.

Problem (On the probability of staying below a threshold function). Let $r(x), x>0$ be a given deterministic function which plays the role of a barrier. Let also the time scale $c>0$ be sufficiently large so that the rescaled process $\{\tilde{a}(c) X(c t)\}_{t>0}($ defined in Section 3) is well approximated by the process $\{M(t)\}_{t>0}=\{A(E(t))\}_{t>0}$ and so

$$
P\{\tilde{a}(c) X(c t) \leqslant r(t), 0 \leqslant t \leqslant T\} \approx P\{M(t) \leqslant r(t), 0 \leqslant t \leqslant T\} .
$$

Thus, an important practical problem is to evaluate the last probability for various choices of the barrier function $r$ and the time horizon $T$. We need the following technical result.

Lemma 4.3. Let $u(x) \geqslant 0, x \geqslant 0$ be a càdlág function and let $A=\{A(x)\}_{x \geqslant 0}$ be an $\alpha$-Fréchet extremal process with càdlág paths such that $P\{A(1) \leqslant x\}=\mathrm{e}^{-x^{-\alpha}}, x>0$. Then:

(i) For all $y>0$, with probability one, we have

$$
\sup _{0 \leqslant x \leqslant y} u(x) A(x)=\sup _{0 \leqslant x \leqslant y} u^{*}(x ; y) A(x)
$$

where $u^{*}(x ; y):=\sup _{x \leqslant z \leqslant y} u(z)$.

(ii) For all $y>0$, we have

$$
\sup _{0 \leqslant x \leqslant y} u(x) A(x) \stackrel{d}{=}\left(\int_{0}^{y} u^{*}(x ; y)^{\alpha} \mathrm{d} x\right)^{1 / \alpha} A(1),
$$

where if the last integral is infinite so is the left-hand side, almost surely.

Proof. Part (i) follows easily from monotonicity considerations. Indeed, observe that with probability one, $\sup _{0 \leqslant \tau \leqslant x} A(\tau)=A(x)$, for all $0 \leqslant x \leqslant y$. Thus, the left-hand side of (4.5) equals

$$
\sup _{0 \leqslant x \leqslant y} u(x) \sup _{0 \leqslant \tau \leqslant x} A(\tau)=\sup _{0 \leqslant \tau \leqslant y} A(\tau) \sup _{\tau \leqslant x \leqslant y} u(x)
$$

where the last relation follows from interchanging the two suprema. This completes the proof of (4.5). Note that since $u$ is càdlág then so is $x \mapsto u^{*}(x ; y)$, and observe that $u^{*}(x ; y)$ is non-increasing in $x$.

We now turn to proving (ii) and suppose first that $\int_{0}^{y} u^{*}(x ; y)^{\alpha} \mathrm{d} x<\infty$. In view of (i), we can assume $u(x)$ to be non-increasing and hence $u(x)=u^{*}(x ; y)$. To prove (ii), approximate $u(x)$ below and above as follows:

$$
u_{L, n}(x)=\sum_{k=1}^{2^{n}} u\left(y k / 2^{n}\right) \rrbracket_{\left[y(k-1) / 2^{n}, y k / 2^{n}\right)}(x)
$$


and

$$
u_{U, n}(x)=\sum_{k=1}^{2^{n}} u\left(y(k-1) / 2^{n}\right) \rrbracket_{\left[y(k-1) / 2^{n}, y k / 2^{n}\right)}(x) .
$$

Thus,

$$
u_{L, n}(x) \leqslant u(x) \leqslant u_{U, n}(x) \text { for all } 0<x \leqslant y, \quad n \in \mathbb{N} .
$$

The result is valid for the càdlág step-functions $u_{L, n}$ and $u_{U, n}$. Indeed, by the independence and the stationarity of the maxincrements of $\{A(x)\}_{x} \geqslant 0$, we have

$$
\left\{A\left(y k / 2^{n}\right), 1 \leqslant k \leqslant 2^{n}\right\} \stackrel{d}{=}\left\{\left(y / 2^{n}\right)^{1 / \alpha} \max _{1 \leqslant i \leqslant k} Z_{i}, 1 \leqslant k \leqslant 2^{n}\right\},
$$

where $\left(Z_{i}\right)$ are i.i.d. standard $\alpha$-Fréchet variables $P\left\{Z_{1} \leqslant x\right\}=\mathrm{e}^{-x^{-\alpha}}, x>0$. Thus

$$
\begin{aligned}
\sup _{0 \leqslant x \leqslant y} u_{L, n}(x) A(x) & \stackrel{d}{=}\left(y / 2^{n}\right)^{1 / \alpha} \max _{1 \leqslant k \leqslant 2^{n}} u\left(y k / 2^{n}\right) \max _{1 \leqslant i \leqslant k} Z_{i} \\
& =\left(y / 2^{n}\right)^{1 / \alpha} \max _{1 \leqslant i \leqslant 2^{n}}\left(\max _{i \leqslant k \leqslant 2^{n}} u\left(y k / 2^{n}\right)\right) Z_{i} \\
& =\left(y / 2^{n}\right)^{1 / \alpha} \max _{1 \leqslant i \leqslant 2^{n}} u\left(y i / 2^{n}\right) Z_{i} \stackrel{d}{=}\left(\sum_{i=1}^{2^{n}} u\left(y i / 2^{n}\right)^{\alpha} y / 2^{n}\right)^{1 / \alpha} Z_{1},
\end{aligned}
$$

where in the last two relations we used the monotonicity of the function $u$ and the max-stability property of the independent Fréchet variables $\left(Z_{i}\right)$, i.e., the fact that $\max \left\{c_{1} Z_{1}, c_{2} Z_{2}\right\} \stackrel{d}{=}\left(c_{1}^{\alpha}+c_{2}^{\alpha}\right)^{1 / \alpha} Z_{3}$. (see, e.g., Embrechts et al., 1997; Resnick, 1987). Observe that the sum in the right-hand side of (4.7) equals $\int_{0}^{y} u_{L, n}(x)^{\alpha} \mathrm{d} x$, which implies (4.6) with $u$ replaced by $u_{L, n}$.

Since $u(x) \geqslant 0$ is non-decreasing, one can show, by using the fact that $u_{L, n}(x)$ and $u_{U, n}(x)$ differ by more than $\varepsilon$, over at most $u(y) / \varepsilon$ number of the $2^{n}+1$ intervals in the partition $\left\{k y / 2^{n}\right\}_{k=1}^{2^{n}}$, that

$$
\lim _{n \rightarrow \infty} \int_{0}^{y} u_{L, n}(x)^{\alpha} \mathrm{d} x=\int_{0}^{y} u(x)^{\alpha} \mathrm{d} x=\lim _{n \rightarrow \infty} \int_{0}^{y} u_{U, n}(x)^{\alpha} \mathrm{d} x .
$$

One has moreover that

$$
\xi_{n}:=\sup _{0 \leqslant x \leqslant y} u_{L, n}(x) A(x) \leqslant \sup _{0 \leqslant x \leqslant y} u(x) A(x) \leqslant \sup _{0 \leqslant x \leqslant y} u_{U, n}(x) A(x)=: \eta_{n}
$$

almost surely. Note that $\xi_{n} \leqslant \xi_{n+1}$ and $\eta_{n+1} \leqslant \eta_{n}$, almost surely, for all $n \in \mathbb{N}$, and observe that the $\xi_{n}$ 's and $\eta_{n}$ 's are $\alpha$-Fréchet variables with scale coefficients converging to $\sigma:=\left(\int_{0}^{y} u(x)^{\alpha} \mathrm{d} x\right)^{1 / \alpha}$. Therefore, $\xi_{n}$ and $\eta_{n}$ both converge almost surely to the same $\alpha$-Fréchet variable $\xi=\sup _{0 \leqslant x \leqslant y} u(x) A(x)$ which has scale coefficient $\sigma$. This yields (4.6) and completes the proof in the case when $\int_{0}^{y} u^{*}(x ; y)^{\alpha} \mathrm{d} x<\infty$. If the last integral is infinite, then one can similarly show by using the lower bound in (4.8) that the left-hand side of (4.6) is infinite, almost surely.

We can now address the barrier problem formulated above. We consider the case of a heavy-tailed extremal process $\{A(x)\}_{x} \geqslant 0$, which is of primary interest in our applications, that is, $P\{A(1) \leqslant x\}=\mathrm{e}^{-x^{-\alpha}}, x>0$. Let $r(x)>0$ be a positive barrier and observe that

$$
P\{A(x) \leqslant r(x), 0 \leqslant x \leqslant y\}=P\left\{\sup _{0 \leqslant x \leqslant y} A(x) / r(x) \leqslant 1\right\} .
$$

Thus, the last probability can be evaluated by using Lemma 4.3 with $u(x):=1 / r(x)$. Since Relation $(4.6)$ is in terms of $u^{*}(x ; y)=$ $\left(\inf _{x \leqslant z \leqslant y} r(z)\right)^{-1}$, which is non-increasing, without loss of generality, and for simplicity, we will assume that the barrier $r(x)$ is non-decreasing, and thus $u^{*}(x ; y)=1 / r(x)$. Now, by using the independence of the processes $\{E(t)\}_{t \geqslant 0}$ and $\{A(x)\}_{x} \geqslant 0$ we obtain the following formula.

Proposition 4.4. Let $r(t)>0, t \geqslant 0$ be a positive, non-decreasing and continuous barrier function. Under the above assumptions on the extremal process $\{A(x)\}_{x \geqslant 0}$ and the process $\{E(t)\}_{t \geqslant 0}$, we have

$$
P\{A(E(t)) \leqslant r(t), 0 \leqslant t \leqslant T\}=\mathbb{E} \exp \left\{-\int_{0}^{E(T)} r^{-\alpha}(D(x)) \mathrm{d} x\right\},
$$


where the right-hand side is interpreted as zero if the integral diverges. In particular, for $T=\infty$, we obtain the probability that $M(t)=A(E(t))$ never goes above the barrier $r$ is: $\mathbb{E} \exp \left\{-\int_{0}^{\infty} r^{-\alpha}(D(x)) \mathrm{d} x\right\}$. Here $\{D(x)\}_{x} \geqslant 0$ is a $\beta$-stable subordinator and $\{E(t)\}_{t} \geqslant 0$ is its inverse process.

Proof. Observe that, since $E(t)$ has almost surely continuous sample paths, in view of $(2.8)$ we have $A(E(t)) \leqslant y r(t)$ for all $0 \leqslant t \leqslant T$ if and only if $A(x) \leqslant y r(t(x))$ for all $0 \leqslant x \leqslant E(T)$, where $t(x)=\sup \{t: E(t) \leqslant x\}=\sup \{t: D(x) \geqslant t\}=D(x)$. Hence

$$
\sup _{0 \leqslant t \leqslant T} r^{-1}(t) A(E(t))=\sup _{0 \leqslant x \leqslant E(T)} r^{-1}(D(x)) A(x) \text { almost surely. }
$$

Thus, in view of Lemma 4.3(ii), the left-hand side of (4.9) equals

$$
P\left\{\sup _{0 \leqslant x \leqslant E(T)} r^{-1}(D(x)) A(x) \leqslant 1\right\}=\mathbb{E} \exp \left\{-\int_{0}^{E(T)} r^{-\alpha}(D(x)) \mathrm{d} x\right\},
$$

where in the last relation, we used the independence of $\{A(x)\}$ and $\{D(x)\}$.

The result for an infinite time horizon follows from the Lebesgue dominated convergence theorem by applying (4.9) for $T=T_{n} \uparrow \infty$ as $n \rightarrow \infty$.

\section{Finite mean waiting times: two-scale asymptotics}

Here, following the ideas of Becker-Kern et al. (2003), we address the case when the $J_{i}$ 's have a finite mean $\mu$ but infinite variance. We prove a functional limit theorem for the process $X^{(c)}(t)=M_{Y}\left(N_{t}^{(c)}\right)$, where now $N_{t}^{(c)}$ models the arrival process of the $J_{i}$ 's on two asymptotic scales $c$ and $b(c)^{-1}=\mathrm{o}(c)$ as $c \rightarrow \infty$. The limit process is the extremal process $\{A(t)\}$ with its time index replaced by the (left-continuous) inverse of the supremum of a Lévy stable motion with a drift.

We suppose that the $J_{i}$ 's are non-negative and that they belong to the sum-domain of attraction of a $\beta$-stable distribution

$$
P\left\{J_{1}>x\right\} \sim x^{-\beta} L(x) \text { as } x \rightarrow \infty \text { where now } 1<\beta<2 \text {, }
$$

for a slowly varying function $L$. Then $\mu=\mathbb{E} J_{i}<\infty$, and for a suitable $b(c)$, as $c \rightarrow \infty$,

$$
\left\{b(c) \sum_{i=1}^{[c t]}\left(J_{i}-\mu\right)\right\}_{t \geqslant 0} \stackrel{f d d}{\Longrightarrow}\{D(t)\}_{t \geqslant 0}
$$

where the limit $D=\{D(t)\}$ is a strictly $\beta$-stable process (totally skewed to the right). Since $\beta \in(1,2)$, the process $D$ can take negative values with positive probability and it is not a subordinator. Nevertheless, this case is of interest in applications and it can be handled by using the two-scale limit regime introduced in Becker-Kern et al. (2003). Indeed, let

$$
T^{(c)}(n)=\sum_{i=1}^{n}\left(c^{-1} \mu+b(c)\left(J_{i}-\mu\right)\right)
$$

and observe that the times $T^{(c)}(n)$ involve two asymptotic scales: a fast scale $c$ focusing on the mean $\mu$ of the $J_{i}$ 's and a slow scale $b(c)^{-1}=\mathrm{o}(c)$ (since $b(c)^{-1}$ varies regularly with index $1 / \beta<1$ ) which focuses on the fluctuations of the $J_{i}$ 's about their mean. Since the rescaled $T^{(c)}(n)$ 's are not necessarily ordered in $n$, we let as in Becker-Kern et al. (2003),

$$
\tau_{n}^{(c)}=\max \left\{T^{(c)}(j): 0 \leqslant j \leqslant n\right\} \text { and set } N_{t}^{(c)}=\inf \left\{n \geqslant 0: \tau_{n}^{(c)} \geqslant t\right\}
$$

for $t \geqslant 0$. Thus $\tau_{n}^{(c)}, n=1,2, \ldots$ represent the arrival times and $N_{t}^{(c)}$ is the number of events by time $t$ in the two scales regime. By Becker-Kern et al. (2003, Theorem 3.1), we have

$$
\left\{c^{-1} N_{t}^{(c)}\right\}_{t \geqslant 0} \stackrel{f d d}{\Longrightarrow}\{\bar{E}(t)\}_{t \geqslant 0} \quad \text { as } c \rightarrow \infty
$$

where $\{\bar{E}(t)\}$ is the left-continuous inverse of the process

$$
D^{*}(t)=\sup \{\mu u+D(u): 0 \leqslant u \leqslant t\}
$$

that is, $\bar{E}(t)=\inf \left\{x \geqslant 0: D^{*}(x) \geqslant t\right\}$. Here $D^{*}(t)$ is the supremum of the $\beta$-stable Lévy motion with a drift: $\{\mu t+D(t)\}$. The drift $\mu t$ corresponds to the 'fast' scale regime of the $T^{(c)}(n)$ 's and the stochastic component $D(t)$ to the 'slow' scale of the fluctuations 
$b(c)\left(J_{i}-\mu\right)$. More precisely, one has $\left\{T^{(c)}([c t])\right\}_{t \geqslant 0} \Rightarrow\{\mu t+D(t)\}_{t \geqslant 0}$ as $c \rightarrow \infty$, in the Skorohod $J_{1}$-topology, thus $\left\{\tau_{[c t]}^{(c)}\right\}_{t} \geqslant 0 \Rightarrow$ $\left\{D^{*}(t)\right\}_{t \geqslant 0}$ in the $J_{1}$-topology (see the proof of Becker-Kern et al., 2003, Theorem 3.1).

Theorem 5.1. Let $\left\{M_{Y}(t)\right\}$ be as in (2.13) and assume that (2.14) holds. We have, as $c \rightarrow \infty$,

$$
\left\{a(c)\left(M_{Y}\left(N_{t}^{(c)}\right)-d(c)\right)\right\}_{t>0} \stackrel{f d d}{\Longrightarrow}\{A(\bar{E}(t))\}_{t>0} .
$$

Proof. We follow closely the proof of Theorem 4.1 in Becker-Kern et al. (2003). Let

$$
\rho_{c}=P_{\left(c^{-1} N_{t_{i}}^{(c)}: 1 \leqslant i \leqslant d\right)} \text { and } \quad \rho=P_{\left(\bar{E}\left(t_{i}\right): 1 \leqslant i \leqslant d\right)}
$$

so that $\rho_{c} \Rightarrow \rho$ as $c \rightarrow \infty$ by (2.11), where $P_{\left(X_{i}: 1 \leqslant i \leqslant d\right)}$ denotes the probability distribution of the vector $\left(X_{i}\right)_{i=1}^{d}$. Let $\mathbf{x} \in \mathbb{R}_{+}^{d}$, $\mathbf{x}=\left(x_{i}\right)_{i=1}^{d}$,

$$
\mu_{c}(\mathbf{x})=P_{\left(a(c)\left(M_{Y}\left(c x_{i}\right)-d(c)\right): 1 \leqslant i \leqslant d\right)} \quad \text { and } \quad v(\mathbf{x})=P_{\left(A\left(x_{i}\right): 1 \leqslant i \leqslant d\right)} .
$$

A transfer theorem, Proposition 4.1 in Becker-Kern et al. (2003), shows that

$$
\int \mu_{c}(x) \mathrm{d} \rho_{c}(x) \Rightarrow \int v(x) \mathrm{d} \rho(x)
$$

so long as $\mu_{c}\left(\mathbf{x}^{(c)}\right) \Rightarrow v(\mathbf{x})$, for all $\mathbf{x}^{(c)} \rightarrow \mathbf{x}$, as $c \rightarrow \infty$. Since (5.3) is equivalent to (5.2), it only remains to check the uniform weak convergence condition.

Let $0<x_{1}<\ldots<x_{d}$ be arbitrary and let $\mathbf{x}^{(c)} \rightarrow \mathbf{x}$ as $c \rightarrow \infty$. For any $y_{1}<\cdots<y_{d}$, we have

$$
P\left\{a(c)\left(M_{Y}\left(c x_{i}\right)-d(c)\right) \leqslant y_{i}, 1 \leqslant i \leqslant d\right\}=F_{Y}\left(y_{1}^{(c)}\right)^{c x_{1}} F_{Y}\left(y_{2}^{(c)}\right)^{c x_{2}-c x_{1}} \cdots F_{Y}\left(y_{d}^{(c)}\right)^{c x_{d}-c x_{d-1}},
$$

where $y_{i}^{(c)}=a(c)^{-1} y_{i}+d(c), i=1, \ldots, d$, and where $F_{Y}(y)=P\left\{Y_{1} \leqslant y\right\}$. Hence, by (2.14) since the finite-dimensional distributions of $\{A(t)\}_{t>0}$ have no atoms, we have as $c \rightarrow \infty$

$$
F_{Y}\left(y_{1}^{(c)}\right)^{c x_{1}} F_{Y}\left(y_{2}^{(c)}\right)^{c x_{2}-c x_{1}} \cdots F_{Y}\left(y_{d}^{(c)}\right)^{c x_{d}-c x_{d-1}} \rightarrow F\left(y_{1}\right)^{x_{1}} F\left(y_{2}\right)^{x_{2}-x_{1}} \cdots F\left(y_{d}\right)^{x_{d}-x_{d-1}},
$$

where $F(y)=P\{A(1) \leqslant y\}$. The last convergence is also valid if the $c x_{i}$ 's are replaced by $c x_{i}^{(c)}=c x_{i}+\mathrm{o}(c)$ as $c \rightarrow \infty$. This shows that $\mu_{c}\left(\mathbf{x}^{(c)}\right) \Rightarrow v(\mathbf{x})$, for all $\mathbf{x}^{(c)} \rightarrow \mathbf{x}$, as $c \rightarrow \infty$, which completes the proof of the result.

Remark 5.2. A simple conditioning argument shows that the limit process $A(\bar{E}(t))$ in Theorem 5.1 has distribution function

$$
F(x, t)=P(A(\bar{E}(t)) \leqslant x)=\int_{0}^{\infty} F(x)^{s} G(\mathrm{~d} s, t),
$$

where $F(x)=P(A(1) \leqslant x)$ and $G(s, t)=P(\bar{E}(t) \leqslant s)$ is the distribution function of $\bar{E}(t)$ given by formula (3.2) in Becker-Kern et al. (2003).

Remark 5.3. If $J_{i}$ are i.i.d. in the sum-domain of attraction of a normal distribution, then the results of this section still hold, where now $\bar{E}(t)$ is the inverse or hitting time distribution of a Brownian motion with drift, which has an inverse Gaussian distribution (Baeumer and Meerschaert, 2007 and Tweedie, 1957). In this case, the mixture according to $\bar{E}(t)$ is an asymptotic first-order correction to the approximation $c^{-1} N_{t}^{(c)} \sim t / \mu$ as $c \rightarrow \infty$.

\section{Some lemmas}

Lemma 6.1. Let $\xi=\{\xi(t)\}_{t \in \mathbb{R}}$ be a measurable process and let $\left\{\eta, \eta_{n}, n \in \mathbb{N}\right\}$ be a sequence of random variables, independent of $\xi$. If $\xi$ is continuous in probability and $\eta_{n} \stackrel{P}{\rightarrow} \eta$ as $n \rightarrow \infty$, then $\xi\left(\eta_{n}\right) \stackrel{P}{\rightarrow} \xi(\eta)$ as $n \rightarrow \infty$.

Proof. Without loss of generality we will assume that $\xi$ and the $\eta_{n}$ 's are defined on the probability spaces $\left(\Omega_{1}, \mathscr{F}_{1}, P_{1}\right)$ and $\left(\Omega_{2}, \mathscr{F}_{2}, P_{2}\right)$, respectively. By using the measurability of the process $\xi$, one can show that $\zeta=\xi(\eta)$ and the $\zeta_{n}=\xi\left(\eta_{n}\right)^{\prime}$ are well-defined random variables on the product probability space $(\Omega, \mathscr{F}, P) \equiv\left(\Omega_{1} \times \Omega_{2}, \sigma\left(\mathscr{F}_{1} \times \mathscr{F}_{2}\right), P_{1} \times P_{2}\right)$. 
To show that $\zeta_{n} \stackrel{P}{\rightarrow} \zeta$ as $n \rightarrow \infty$, it is enough to prove that

$$
\mathbb{E}\left(1 \wedge\left|\zeta_{n}-\zeta\right|\right)=\int_{\Omega_{1} \times \Omega_{2}}\left(1 \wedge\left|\xi\left(\omega_{1}, \eta_{n}\left(\omega_{2}\right)\right)-\xi\left(\omega_{1}, \eta\left(\omega_{2}\right)\right)\right|\right) P_{1}\left(\mathrm{~d} \omega_{1}\right) P_{2}\left(\mathrm{~d} \omega_{2}\right) \rightarrow 0,
$$

as $n \rightarrow \infty$, where $a \wedge b=\min \{a, b\}$. By the Fubini-Tonelli Theorem, we have

$$
\mathbb{E}\left(1 \wedge\left|\zeta_{n}-\zeta\right|\right)=\int_{\Omega_{2}} h\left(\eta_{n}\left(\omega_{2}\right), \eta\left(\omega_{2}\right)\right) P_{2}\left(\omega_{2}\right)
$$

where $h\left(\tau_{1}, \tau_{2}\right):=\int_{\Omega_{1}}\left(1 \wedge\left|\xi\left(\omega_{1}, \tau_{1}\right)-\xi\left(\omega_{1}, \tau_{2}\right)\right|\right) P_{1}\left(\mathrm{~d} \omega_{1}\right)$. Since $\{\xi(t)\}$ is continuous in probability, the function $h$ is continuous and $h(\tau, \tau)=0, \tau \in \mathbb{R}$. Now, since $\eta_{n} \stackrel{P}{\rightarrow} \eta$, for any $n_{k} \rightarrow \infty$ there exists a further subsequence $\tilde{n}_{k} \rightarrow \infty$ such that $\eta_{\tilde{n}_{k}} \rightarrow \eta$ almost surely, as $\tilde{n}_{k} \rightarrow \infty$. Thus, the continuity of $h$ and the dominated convergence theorem (note that $\left.0 \leqslant h \leqslant 1\right)$ imply $\mathbb{E}\left(1 \wedge\left|\zeta_{\tilde{n}_{k}}-\zeta\right|\right) \rightarrow 0$ as $\tilde{n}_{k} \rightarrow \infty$.

We have thus shown that every subsequence $n_{k} \rightarrow \infty$ contains a further subsequence $\tilde{n}_{k} \rightarrow \infty$ such that $\mathbb{E}\left(1 \wedge\left|\zeta_{\tilde{n}_{k}}-\zeta\right|\right) \rightarrow 0$ as $\tilde{n}_{k} \rightarrow \infty$. This implies that $\zeta_{n} \stackrel{P}{\rightarrow} \zeta$ as $n \rightarrow \infty$.

Lemma 6.2. Let $D=\{D(x)\}_{x} \geqslant 0$ be a $\beta$-stable subordinator $(0<\beta<1)$ with monotone non-decreasing càdlág paths and Laplace transform $\mathbb{E} \mathrm{e}^{-s D}=\mathrm{e}^{-\Gamma(1-\beta) s^{\beta}}, s \geqslant 0$. Let $\mathscr{M}_{D}(\cdot, \omega)$ be the Lebesgue-Stieltjes measure corresponding to path $D(\cdot, \omega)$ of $D$, defined on the Borel $\sigma-$ algebra $\mathscr{B}_{\mathbb{R}_{+}}$.

Then, $\mathscr{M}_{D}(\cdot)$ is an independently scattered $\beta$-stable random measure on $\left(\mathbb{R}_{+}, \mathscr{B}_{\mathbb{R}_{+}}\right)$with control measure $\mu(\mathrm{d} x)=\Gamma(1-\beta)$ $\cos (\pi \beta / 2) \mathrm{d} x$ and unit skewness intensity function in the sense of Samorodnitsky and Taqqu (1994, Definition 3.3.1).

Proof. By definition, the set-valued functions $\mathscr{M}_{D}(\cdot, \omega)$ are $\sigma$-additive and $\sigma$-finite measures, with probability one. Thus, it remains to only show that:

(i) For any Borel set $B \subset[0, \infty)$ with finite Lebesgue measure $|B|$, we have that $\mathscr{M}_{D}(B)$ is a $\beta$-stable random variable with scale coefficient $\sigma^{\beta}=\Gamma(1-\beta) \cos (\pi \beta / 2)|B|$ and unit skewness.

(ii) For any disjoint Borel sets $B_{1}, \ldots, B_{d} \subset[0, \infty)$, the random variables $\mathscr{M}_{D}\left(B_{1}\right), \ldots, \mathscr{M}_{D}\left(B_{d}\right)$ are independent.

Recall that for any Borel set $B \subset(0, \infty)$, we have

$$
\mathscr{M}_{D}(B)=\inf \left\{\sum_{k=1}^{\infty} \mathscr{M}_{D}\left(a_{k}, b_{k}\right]: B \subset \bigcup_{k=1}^{\infty}\left(a_{k}, b_{k}\right] \subset(0, \infty)\right\} .
$$

By the results of LePage et al. (1981) (see also Samorodnitsky and Taqqu, 1994, Chapter 3), we may suppose that with probability one, for all $t \geqslant 0$,

$$
D(t)=\sum_{t_{i} \leqslant t} \varepsilon_{i}^{-1 / \beta}
$$

where $\left\{\left(t_{i}, \varepsilon_{i}\right)\right\}$ are the points of a Poisson point process in $[0, \infty) \times[0, \infty)$ with the Lebesgue intensity measure $\mathrm{d} t \times \mathrm{d} x$. Note that $D(t)$ is càdlág, with probability one.

Let

$$
\widetilde{\mathscr{M}}_{D}(B, \omega):=\sum_{t_{i}(\omega) \in B} \varepsilon_{i}(\omega)^{-1 / \beta},
$$

for any Borel set $B \subset[0, \infty)$. It is easy to see that, with probability one, $\widetilde{\mathscr{M}}_{D}(\cdot, \omega)$ is a well-defined $\sigma$-finite measure on the Borel sets in $[0, \infty)$.

Observe that $\mathscr{M}_{D}(B, \omega)=\widetilde{\mathscr{M}}_{D}(B, \omega)$, for all $B=\bigcup_{k=1}^{n}\left(a_{k}, b_{k}\right]$, with $0 \leqslant a_{k}<b_{k}$. Thus, with probability one $\mathscr{M}_{D}(\cdot, \omega)$ and $\widetilde{\mathscr{M}}_{D}(\cdot, \omega)$ are $\sigma$-finite measures, which agree on the semiring of sets $\mathscr{C}:=\left\{\bigcup_{k=1}^{n}\left(a_{k}, b_{k}\right], 0 \leqslant a_{k}<b_{k}\right\}$. This, by Caratheodory's extension theorem (see, e.g., Billingsley, 1995, p. 166), implies that with probability one, the two measures agree on the $\sigma$-algebra generated by $\mathscr{C}$ i.e., on all Borel sets in $[0, \infty)$. Therefore, it is enough to show that the measure $\widetilde{\mathscr{M}}_{D}$ satisfies (i) and (ii). Property (ii) is immediate since $\left\{\left(t_{i}, \varepsilon_{i}\right)\right\}$ is a Poisson point process. To prove (i), note that by the homogeneity of the intensity

$$
\sum_{t_{i} \in B} \varepsilon_{i}^{-1 / \beta} \stackrel{d}{=} \sum_{t_{i} \leqslant|B|} \varepsilon_{i}^{-1 / \beta} \stackrel{d}{=}|B|^{1 / \beta} \sum_{k=1}^{\infty} \Gamma_{k}^{-1 / \beta}
$$


where $|B|$ is the Lebesgue measure of the Borel set $B$, and where $0 \leqslant \Gamma_{1} \leqslant \Gamma_{2} \leqslant \cdots$ are the points of a standard Poisson point process on the real line with unit intensity. As in LePage et al. (1981) (see also Samorodnitsky and Taqqu, 1994) one can show that $\sum_{k=1}^{\infty} \Gamma_{k}^{-1 / \beta}$ is a $\beta$-stable random variable with Lévy measure $\phi(t, \infty)=t^{-\beta}$ concentrated on the positive axis $t>0$, and hence (see, e.g., Meerschaert and Scheffler, 2001, Theorem 7.3.5) is totally skewed to the right with scale $\operatorname{coefficient} \Gamma(1-\beta) \cos (\pi \beta / 2)$. This implies $(i)$ for the measure $\widetilde{\mathscr{M}}_{D}$ and completes the proof of the lemma.

Lemma 6.3. Let $\left(J_{i}\right)$ be i.i.d. non-negative random variables such that $P\left(J_{i}>t\right) \sim C t^{-\beta}$ as $t \rightarrow \infty$ for some $0<\beta<1$ and $C>0$. Then $(2.3)$ holds with $b_{n}=(C n)^{-1 / \beta}$ and $D$ is a $\beta$-stable positive random variable with Laplace transform $\mathbb{E e}^{-s D}=\mathrm{e}^{-\Gamma(1-\beta) s^{\beta}}, s \geqslant 0$.

Proof. The proof is standard. By Feller (1971, Section XVII.5) (see also Meerschaert and Scheffler, 2001, Theorem 8.2.16, Corollary 8.2.19, and Remark 8.2.20) we have that $a_{n}\left(J_{1}+\cdots+J_{n}\right) \Rightarrow D$ as $n \rightarrow \infty$ if and only if $n P\left(a_{n} J_{1}>t\right) \sim \phi(t, \infty)$ as $n \rightarrow \infty$, where $\phi$ is the Lévy measure of $D$. It is easy to check that this convergence holds with $b_{n}=(C n)^{-1 / \beta}$ and Lévy measure $\phi(t, \infty)=t^{-\beta}$ concentrated on the positive axis $t>0$. Now compute the Laplace transform of the random variable $D$ from the Lévy representation

$$
\mathbb{E} \mathrm{e}^{-s D}=\exp \left(\int_{0}^{\infty}\left(\mathrm{e}^{-s t}-1\right) \phi(\mathrm{d} t)\right)
$$

following the argument in Meerschaert and Scheffler (2001, p. 264).

\section{Acknowledgments}

We thank Professor Elissaveta Pancheva for useful discussions. We also thank the anonymous referees for their important suggestions, which helped us improve our paper and in particular the proof of Theorem 3.1.

\section{References}

Baeumer, B., Meerschaert, M., 2001. Stochastic solutions for fractional Cauchy problems. Fractional Calculus Appl. Anal. 4, 481-500.

Baeumer, B., Meerschaert, M.M., 2007. Fractional diffusion with two time scales. Physica A 373, 237-251.

Baeumer, B., Meerschaert, M.M., Benson, D.A., Wheatcraft, S.W., 2001. Subordinated advection-dispersion equation for contaminant transport. Water Resour. Res. $37,1543-1550$.

Barabási, A.-L., 2005. The origin of bursts and heavy tails in human dynamics. Lett. Nature 435, 207-211.

Becker-Kern, P., Meerschaert, M.M., Scheffler, H.P., 2003. Limit theorem for continuous time random walks with two time scales. J. Applied Probab. 41, $455-466$. Bening, V.E., Korolev, V.Yu., 2002. Generalized Poisson Models and their Applications in Insurance and Finance Modern Probability and Statistics. VSP, Utrecht. Benson, D., Wheatcraft, S., Meerschaert, M., 2000. Application of a fractional advection-dispersion equation. Water Resour. Res. 36, $1403-1412$.

Benson, D., Schumer, R., Meerschaert, M., Wheatcraft, S., 2001. Fractional dispersion, Lévy motions, and the MADE tracer tests. Transport Porous Media 42, $211-240$.

Benson, D.A., Schumer, R., Meerschaert, M.M., 2007. Recurrence of extreme events with power-law interarrival times. Geophys. Res. Lett. 34, L16404-L16408. Berhondo, A.L.M., Taboada, R.E.R., Larralde, L.A., 2006. Waiting time distribution of emissions in complex coronal mass ejections. Astrophys. Space Sci. 302, $213-216$.

Bertoin, J., 1996. Lévy Processes. Cambridge University Press, Cambridge.

Billingsley, P., 1995. Probability and Measure. Wiley, New York.

Bingham, N.H., 1971. Limit theorems for occupation times of Markov processes. Z. Warsch. verw. Geb. 17, 1-22.

Bondesson, L., Kristiansen, G., Steutel, F., 1996. Infinite divisibility of random variables and their integer parts. Statist. Probab. Lett. $28,271-278$.

Brockmann, D., Hufnagel, L., Geisel, T., 2006. The scaling laws of human travel. Nature 439, 462-465.

Caputo, M., 1967. Linear models of dissipation whose Q is almost frequency independent, Part II. Geophys. J. Roy. Astron. Soc. 13, $529-539$.

Cheng, B., Epstein, R.I., Guyer, R.A., Young, A.C., 1995. Earthquake-like behavior of soft $\gamma$-ray repeaters. Nature 382, 518-520.

Dwass, M., 1964. Extremal processes. Ann. Math. Statist. 35 (4), 1718-1725.

Embrechts, P., Klüppelberg, C., Mikosch, T., 1997. Modelling Extreme Events. Springer, New York.

Feller, W., 1971. An Introduction to Probability Theory and its Applications, second ed., vol. II. Wiley, New York.

Korolev, V.Yu., 1994. Convergence of random sequences with independent random indices I. Theory Probab. Appl. 39, $282-297$ (English translation).

Korolev, V.Yu., 1995. Convergence of random sequences with independent random indices II. Theory Probab. Appl. 40, 770-772 (English translation).

Kruglov, V.M., Korolev, V.Yu., 1990. Limit Theorems for Random Sums. Moscow State University Publishing House, Moscow. (in Russian).

Lamperti, J., 1964. On extreme order statistics. Ann. Math. Statist. 35, 1726-1737.

Lavergnat, J., Golé, P., 1998. A stochastic raindrop time distribution model. J. Appl. Meteorol. 37, 805-818.

Leadbetter, M.R., Lindgren, G., Rootzén, H., 1983. Extremes and Related Properties of Random Sequences and Processes. Springer, New York.

LePage, R., Woodroofe, M., Zinn, J., 1981. Convergence of a stable distribution via order statistics. Ann. Probab. 9, 624-632.

Mainardi, F., Gorenflo, R., 2000. On Mittag-Leffer-type functions in fractional evolution processes. J. Comput. Appl. Math. 118, $283-299$.

Mainardi, F., Raberto, M., Gorenflo, R., Scalas, E., 2000. Fractional calculus and continuous-time finance II: the waiting-time distribution. Phys. A 287, 468-481. Meerschaert, M., Scheffler, H.P., 2001. Limit Distributions for Sums of Independent Random Vectors. Wiley, New York.

Meerschaert, M., Benson, D., Scheffler, H.P., Baeumer, B., 2002. Stochastic solution of space-time fractional diffusion equations. Phys. Rev. E 65, $1103-1106$.

Meerschaert, M.M., Scheffler, H.P., 2004. Limit theorems for continuous time random walks with infinite mean waiting times. J. Appl. Probab. 41 (3), 623-638.

Meerschaert, M.M., Scheffler, H.P., 2008. Triangular array limits for continuous time random walks. Stochastic Process. Appl. 118 (9), $1606-1633$.

Metzler, R., Klafter, J., 2004. The restaurant at the end of the random walk: recent developments in the description of anomalous transport by fractional dynamics. J. Phys. A 37, R161-R208.

Miller, K., Ross, B., 1993. An Introduction to the Fractional Calculus and Fractional Differential Equations. Wiley, New York.

Pancheva, E.I., 1998. Self-similar extremal processes. in: Proceedings of the 18th Seminar on Stability Problems for Stochastic Models Part I, Hajdúszoboszló, 1997. J. Math. Sci. (New York) 92 (3), 3911-3920. 
Pancheva, E.I., Jordanova, P.K., 2004. Functional transfer theorems for maxima of iid random variables functional transfer theorems for maxima of iid random variables. Dokl. Balgaskata Akad. Nauk. C.R. Acad. Bulgare Sci. 57 (8), 9-14.

Pancheva, E.I., Mitov, I., Volkovich, Z., 2006a. Sums and extremal processes over explosion area. Dokl. Balgaskata Akad. Nauk. C.R. Acad. Bulgare Sci. 57 (8), 9-14. Pancheva, E.I., Kolkovska, E.T., Jordanova, P.K., 2006b. Random time-changed extremal processes. Teor. Veroyatn. Primen. 59 (12), $1219-1226$ (Translated in Theory Probab. Appl.).

Pancheva, E., Volkovich, Z., Morozensky, L., 2007. Upper and lower bounds for ruin probability. Pliska Stud. Math. Bulgar. 18, $315-326$.

Podlubny, I., 1999. Fractional Differential Equations. Academic Press, New York.

Resnick, S.I., 1974. Inverses of extremal processes. Adv. in Appl. Probab. 6, 392-406

Resnick, S.I., 1975. Weak convergence to extremal processes. Ann. Probab. 3, 951-960.

Resnick, S.I., 1986. Point processes, regular variation, and weak convergence. Adv. in Appl. Probab. 18 (1), 66-138.

Resnick, S.I., 1987. Extreme Values, Regular Variation and Point Processes. Springer, New York.

Resnick, S.I., Rubinovitch, M., 1973. The structure of extremal processes. Adv. in Appl. Probab. 5, 287-307.

Resnick, S.I., Stărică, C., 1995. Consistency of Hill's estimator for dependent data. J. Appl. Probab. 32, 139-167.

Sabatelli, L., Keating, S., Dudley, J., Richmond, P., 2002. Waiting time distributions in financial markets. Eur. Phys. J. B 27, $273-275$.

Samko, S., Kilbas, A., Marichev, O., 1993. Fractional Integrals and Derivatives: Theory and Applications. Gordon and Breach, London.

Samorodnitsky, G., Taqqu, M., 1994. Stable non-Gaussian Random Processes. Chapman \& Hall, New York.

Sato, K.I., 1999. Lévy Processes and Infinitely Divisible Distributions. Cambridge University Press, Cambridge.

Scalas, E., 2006. Five years of continuous-time random walks in econophysics. The Complex Networks of Economic Interactions, Lecture Notes in Economics and Mathematical Systems, vol. 567, Springer, Berlin, pp. 3-16.

Schumer, R., Benson, D.A., Meerschaert, M.M., Wheatcraft, S.W., 2001. Eulerian derivation of the fractional advection-dispersion equation. J. Contaminant Hydrol. $48,69-88$.

Seneta, E., 1976. Regularly Varying Functions. Lecture Notes in Mathematics, vol. 508, Springer, Berlin.

Silvestrov, D.S., 2004. Limit Theorems for Randomly Stopped Stochastic Processes. Springer Series in Statistics. Probability and its Applications, Springer, New York.

Silvestrov, D.S., Teugels, J.L., 1998. Limit theorems for extremes with random sample size. Adv. in Appl. Probab. 30, 777-806.

Silvestrov, D.S., Teugels, J.L., 2004. Limit theorems for mixed max-sum processes with renewal stopping. Ann. Appl. Probab. 14, $1838-1868$.

Smethurst, D.P., Williams, H.C., 2001. Are hospital waiting lists self-regulating? Nature 410, 652-653.

Tweedie, M.C.K., 1957. Statistical properties of inverse Gaussian distributions I. Ann. Math. Statist. 28, 362-377. 